\title{
Application of Moire in Modern Dress
}

\author{
Mengyuan Zheng ${ }^{1, *}$ \\ ${ }^{1}$ College of Art and Design, Wuhan Textile University, Wuhan, Hubei, China \\ *Corresponding author. Email: 1119718124@qq.com
}

\begin{abstract}
Moire is one of China's classic traditional patterns. Now the modern redesign of traditional patterns becomes more and more popular. This article will explore the artistic value of moire in traditional costumes, provide a more favorable theoretical basis for modern design, and trigger people's artistic thinking about traditional moire. This paper will use literature review, market research and other research methods to analyze the modeling characteristics of moire, and explore the application form of moire in modern clothing. This article will also elaborate the origin and development of moire, the deformation design of moire, and the application of moire in modern clothing.
\end{abstract}

Keywords: Cloud pattern, Clothing, Traditional pattern.

\section{INTRODUCTION: ORIGIN AND DEVELOPMENT OF MOIRE}

In the history of 5000 years of Chinese civilization, moire is one of China's traditional patterns, changing and developing in the long river of the times. The shape and beautiful meaning of Moire are all the enrichment and refinement of Chinese excellent culture. Nowadays, more and more people begin to advocate the development of excellent traditional culture, and the classic pattern of cloud pattern has naturally become one of the focuses of designers all over the world. Chinese traditional culture pays attention to the expression of "both spirit and shape", but many of the redesign of moire in modern design works are based on the change of moire shape, which do not pay attention to the transmission of its spirit. The beauty of cloud pattern lies not only in its shape, but also in the Chinese aesthetic understanding and concept of cloud culture. This paper will comb the development of moire, analyze the feasibility of Moire redesign, and explore the application of moire in clothing.

\subsection{Origin of Moire}

In ancient China, the ecological model of smallscale peasant economy was destined to inextricably link with the weather. The clouds rose, evaporated and condensed into rain to moisten everything.
"ShuoWenJieZi" contains: "mountains and rivers are natural, from rain to clouds." It shows that there is an inseparable relationship between cloud and rain. The origin of cloud pattern is always one of the most difficult problems in the history of Chinese pattern. It is not difficult to see the shadow of cloud pattern from the spiral pattern of Neolithic painted pottery. However, whether it had the meaning of cloud at that time cannot be studied now. It is not difficult to infer that the origin of moire is influenced by many factors. Some people think that the cloud pattern originated from the pictographic generalization of natural things. In primitive society, people found inspiration in nature, such as the snail shell, the vortex of flowing water, and the shape of vortex extracted from the vine of plants. This is not a simple imitation of the ancestors, but a consensus reached with the form of nature through conscious thinking, so as to extract a purely regular geometric pattern. In terms of form alone, it seems that it is not inappropriate to define moire as the abstraction and generalization of natural things. However, the standard to measure the origin of moire is not only by form.

\subsubsection{The Theory of Technology Caused by Labor}

Some people think that the spiral pattern is the origin of moire. In the process of laboring, people observed the process of mixing soil, making 
porcelain and spinning on grinding wheel, and connected it with the spiral pattern in natural phenomena to create the structure of spiral pattern. In addition, due to the particularity of the human body, it is not difficult to make the rotation action. It is not difficult for human wrist to drive the tool to rotate, so that the spiral pattern is easy to be accepted by human both visually and by sensory.

\subsubsection{Worship Theory Caused by Belief}

Some people think that moire originated from worship of faith. In ancient society, people often hold sacrificial ceremonies for harvest. "Cloud" and "rain" are inseparable. It is not difficult to see that people prefer to believe that cloud and rain are closely related. In "Zuo Zhuan", it is recorded that "the emperors of the past were named after the cloud age, and then the cloud master". The ancients compared Huang Di with Yun Xiang, which is enough to see the importance of cloud culture. In the ancient people's consciousness, cloud can bring good luck and harvest, so their worship of cloud also arises spontaneously. The rising form of cloud disk is very mysterious, and it has become a very expressive symbol in sacrifice.

\subsection{The Development of Moire}

Different periods of cloud decoration is not the same, it has been given new forms in the long history. When studying moire, scholars must understand its different modeling characteristics in each period, which is convenient to grasp the changing law of moire and the connotation of moire.

\subsubsection{The Budding Period of Moire Pattern}

Following the origin of the cloud pattern, we can find a trace of connection between the cloud and thunder patterns of Shang and Zhou bronzes and the spiral patterns of painted pottery. Xu Wen once mentioned in Chinese cloud pattern decoration: "in the sense of morphology, the origin of Chinese cloud pattern is inextricably linked with the spiral pattern in geometric pattern." However, the origin of swirling pattern as moire is always lack of research, so we can not directly regard swirling pattern as the origin of moire. Finally, the cloud and thunder patterns on the bronzes of the last week period were regarded as the early forms of the cloud pattern.

\subsubsection{The Development Period of Moire Pattern}

The cloud pattern of Han Dynasty has been developed unprecedentedly. During this period, the cloud pattern has evolved into cirrus pattern, cloud Qi pattern and cloud beast pattern with the characteristics of the times. The content of cloud pattern is more abundant and the form is more diverse. In Mawangdui Han Tomb, jewelry and clothing unearthed can see the figure of moire.

\subsubsection{The Mature Period of Moire}

Sui and Tang Dynasties is a period of great integration of economy and culture. The introduction of Buddhism into the Central Plains had a great impact on the form of cloud pattern. During this period, flower moire with the characteristics of the times was formed in Tang Dynasty, and the formation of flower moire opened up a new pattern of the development of moire. Since then, the Song Yun, Ming and Qing Dynasties' regiment cloud pattern, Ruyi Yun and stacked cloud pattern have inherited and developed the cloud pattern in the Tang Dynasty.

\section{DEFORMATION DESIGN OF MOIRE}

\subsection{Simplified Design of Moire}

In a modern society that emphasizes simplicity and fashion, if designers want to better apply moire to clothing, they need to simplify the design and extract the essence to better meet the aesthetic standards of modern people. In the design process, the complicated moire can be simplified, the classic moire model can be retained, and the unnecessary decorative elements can be deleted at the same time, and more attention should be paid to the abstract expression of moire. Through the redesign of moire modeling, the pattern is extracted to the appropriate carrier, and these materials are reasonably arranged to make the whole modeling of Moire plumper, and the redundant components are deleted to make the overall modeling of Moire more concise. In the process of design, designers should also pay attention to the relationship between various elements in moire to maintain the integrity of the work. 


\subsection{Deconstruction Design of Moire}

Pattern decomposition, deformation and reconstruction are not uncommon in the application of clothing. Through the deconstruction design of moire, it has more characteristics of the times and better integrates into the current scene. In the deformation design of moire, select the appropriate axis arrangement and reorganization in the layout, scatter and reorganization in the direction, and redesign the moire by using deformation, stretching, distortion and other methods. This design is no longer a copy and paste of the traditional pattern, but injects the moire into the soul of the new era. As everyone knows, the design that can reflect the characteristics of an era is the design that can be accepted by people. The traditional and single pattern cannot meet the growing spiritual needs of people. Therefore, it is the general trend to decompose and reorganize the traditional moire form, select the most representative characteristics of moire, and use the form of midpoint, line and surface of plane composition to carry out secondary design, take abstract expression as a breakthrough, abandon the realistic techniques in traditional pattern creation, and design new moire patterns that conform to modern aesthetics and have cultural connotation. The deconstruction design of moire is not only a breakthrough in the modeling of moire pattern, but also a recreation of traditional pattern. It is a new symbol with the characteristics of the times, and is more easily accepted by the public on the basis of carrying forward the traditional culture of the Chinese nation.

\subsection{Restructuring Design of Moire}

In the process of fashion design, design and technology are inseparable. The reorganization design of moire should consider not only the integrity of its own pattern, but also the effect it presents in the clothing. Design works should consider the current environment, source of inspiration, clothing style, fabric mechanism, color expression and other factors. The characteristics of fabric should be taken into account in the redesign of moire. Traditional moire patterns are mostly presented in the form of embroidery and brocade. Nowadays, with the continuous progress of science and technology, the continuous improvement of modern tie dyeing technology, the continuous development of digital printing and jacquard technology has brought new possibilities for the expression of moire in clothing. Designers are looking for new ways to constantly try and break through in the pattern form and process method, trying to bring new visual experience to the audience, such as adding moire decoration in the hem, neckline, placket and other places of leisure wear. Using simple and atmospheric colors can better meet the aesthetic taste of the times, and bring new breakthroughs for the secondary design of moire.

\section{APPLICATION OF MOIRE IN MODERN FASHION DESIGN}

With the development of science and technology, it brings new possibilities for the application of moire in clothing and new opportunities for the development of traditional culture. In the 2008 Beijing Olympic Games and the 2014 APCE conference, people can see the shadow of moire, which shows that the upsurge of using moire in clothing is becoming more and more intense. The application of cloud pattern to clothing can not only reflect people's inheritance and development of traditional culture, but also show the national pride of Chinese people.

\subsection{The Breakthrough of Modeling}

Moire has very high aesthetic value and beautiful meaning. The progress of science and technology has brought a new breakthrough for people's aesthetic level. While conforming to the western trend, designers should also pay attention to carrying forward our nation's excellent culture. Moire pattern has formed a huge system in the rolling history. In order to make a breakthrough in moire pattern, designers should first understand the historical background behind moire pattern, and refine and sublimate the pattern through the abstract method. In the creation, the creators cannot copy the achievements of predecessors step by step. They should deeply analyze the content involved, understand the characteristics of each kind of moire and the cultural implication of its era, and constantly adjust and improve it in the actual design process, so as to design excellent works.

\subsection{Sublimation of Moral}

The history of fashion design in China started late, and has been deeply influenced by western design in the form, concept and implication of fashion design. It is not only the historical mission of every Chinese designer, but also an urgent task of the times to carry forward Chinese traditional 
culture. In order to win China's discourse power on the international fashion stage, designers should pay attention to the connection between Chinese excellent traditional culture and international trends, and constantly absorb and draw lessons from excellent design while inheriting Chinese traditional culture, so as to create design styles with Chinese characteristics. Moire is one kind of China's traditional classical pattern. Its reorganization and innovation is a breakthrough and continuation of China's traditional culture. Due to the diversity of moire patterns, it provides new possibilities for its innovation in clothing, lays a solid foundation for the inheritance and development of Chinese culture, and provides a broad space for the development of modern fashion design on the international stage.

\subsection{The Innovation of Color}

The reasonable collocation of colors can make the finishing point for fashion design. The traditional moire patterns are mainly red, yellow, green, black and white. The redesign of moire should not only make a breakthrough in modeling, but also cannot ignore the role of color. When extracting the traditional moire modeling, designers should consider whether it conforms to the current design atmosphere. Designers can make a new attempt on the basis of breaking the traditional color system, otherwise it may bring unexpected results.

\section{CONCLUSION}

The modeling characteristics of moire and the aesthetic implication given by people show the powerful vitality of moire. As one of the most basic classic patterns, moire plays an important role in fashion design. It is of practical significance to split, restructure, deform and redesign moire in clothing. It creates new ideas for fashion design, craft design and source of inspiration, and provides new methods for the collision and integration of Chinese and Western cultures. On the basis of a deep understanding of Moire culture, designers should constantly bring forth the new, rationally apply this classic visual symbol, make the excellent culture of the Chinese nation continue to internationalize, and carry forward this visual symbol with Oriental mysterious artistic conception.

\section{AUTHORS' CONTRIBUTIONS}

This article is independently completed by Mengyuan Zheng.

\section{REFERENCES}

[1] Liu Yongru. Reference and application of traditional moire pattern in modern fashion design $[\mathrm{J}]$. Art science and technology, 2018,31 (09): 96-97

[2] Wu Yilun, Pan Ruru, Gao Weidong. Appearance simulation of weft knitted fabric with Moire yarn [J]. Silk, 2019,56 (02): 37-42

[3] Ding Peili, Wang Yarong. The fashion evolution of women's shirts in Ming Dynasty - starting from an unearthed Sihe Ruyi dark flower cloud cloth woman's shirt [J]. Southern cultural relics, 2019 (02): 259-269

[4] Shang Yali. Jixiang cloud pattern and modern home soft decorative textile design [J]. Textile technology progress, 2015 (01): 55-58

[5] Yu Lixia. Research on the evolution of cloud and thunder patterns based on art semiotics [D]. Northwest University, 2019

[6] Yu Wan. The aesthetic characteristics of the bronze tiger button with cloud pattern and Its Enlightenment on modern design $[\mathrm{J}]$. Identification and appreciation of cultural relics, 2019 (16): 86-88

[7] Li Wenjiao. Research on the aesthetics of traditional Chinese moire and its application in modern fashion [D]. Wuhan Textile University, 2018

[8] Wang Zixuan. A thousand forms and all things follow Shu Juan [D]. Shaanxi Normal University, 2018

[9] Miao Min. research on the application of traditional moire pattern in modern design [D]. Zhejiang University of technology, 2017

[10] Wang Yinming, Tao Yingyan. Application of Jixiang cloud pattern in modern fashion design [J]. Textile industry and technology, 2019,48 (09): $55-56$

[11] Zhou Qi. Cloud pattern re modeling design [D]. Zhejiang University of technology, 2018

[12] Gao Na, Li Qiang, Xu Bojun, Liu Xinjin, Wang guangbin. Study on the periodicity of 
Moire fancy yarn [J]. Silk, 2018,55 (04): 2430

[13] Zheng Jiaru. Research on the application of Han Dynasty cloud pattern in the fabric design of modern meditation clothing [D]. Zhejiang University of technology, 2018

[14] Gold plated and silver inlaid bronze tripod [J]. Journal of the National Museum of China, 2018 (02): 2

[15] Li Ying, Wang Le. Research on moire in Han Dynasty textiles $[\mathrm{J}]$. Progress in textile science and technology, 2016 (10): 5-8 